\title{
Mitochondrial Syndromes with Leukoencephalopathies
}

\author{
Lee-Jun C. Wong, Ph.D. ${ }^{1}$ \\ ${ }^{1}$ Department of Molecular and Human Genetics, Baylor College of \\ Medicine, Houston, Texas \\ Semin Neurol 2012;32:55-61.

\begin{abstract}
Address for correspondence and reprint requests Lee-Jun C. Wong, Ph.D., Professor, Department of Molecular and Human Genetics, Baylor College of Medicine, One Baylor Plaza, NAB2015, Houston, TX 77030 (e-mail: ljwong@bcm.edu).
\end{abstract}

Abstract
Keywords
- mitochondrial
leukoencephalopathy
- mitochondrial
disorders
- Leigh's disease
- POLG
- MNGIE
- MPV17
- DARS2

White matter involvement has recently been recognized as a common feature in patients with multisystem mitochondrial disorders that may be caused by molecular defects in either the mitochondrial genome or the nuclear genes. It was first realized in classical mitochondrial syndromes associated with mitochondrial DNA (mtDNA) mutations, such as mitochondrial encephalomyopathy with lactic acidosis and stroke-like episodes (MELAS), Leigh's disease, and Kearns-Sayre's syndrome. Deficiencies in respiratory chain complexes I, II, IV, and V often cause Leigh's disease; most of them are due to nuclear defects that may lead to severe early-onset leukoencephalopathies. Defects in a group of nuclear genes involved in the maintenance of mtDNA integrity may also affect the white matter; for example, mitochondrial neurogastrointestinal encephalomyopathy (MNGIE) caused by thymidine phosphorylase deficiency, Navajo neurohepatopathy (NNH) due to MPV17 mutations, and Alpers syndrome due to defects in DNA polymerase gamma (POLG). More recently, leukoencephalopathy with brainstem and spinal cord involvement and lactate elevation (LBSL) has been reported to be caused by autosomal recessive mutations in a mitochondrial aspartyl-tRNA synthetase, DARS2 gene. A patient with leukoencephalopathy and neurologic complications in addition to a multisystem involvement warrants a complete evaluation for mitochondrial disorders. A definite diagnosis may be achieved by molecular analysis of candidate genes based on the biochemical, clinical, and imaging results.

\section{Background}

Mitochondria are cellular power plants that carry out oxidation of the fuel source, carbohydrates and fatty acids, via tricarboxylic acid (TCA) cycle and fatty acid oxidation, to form NADH and FADH2, which in turn reduce the oxygen molecules by way of respiration through the electron transport chain, and finally generate the ATP energy molecules. Mitochondrial dysfunction results in energy deficiency that affects preferentially the high-energy demand tissues, such as brain and muscle. Because every cell contains hundreds to thousands of mitochondria, and the oxidative energy metabolism is an essential cellular activity, the disease can potentially affect any organ or tissue of an individual at any age with any kind of clinical features. ${ }^{1}$

Issue Theme Inherited

Leukoencephalopathies; Guest Editor, Deborah L. Renaud, M.D.
Mitochondria are cellular organelles made up of gene products encoded by the two genomes, mitochondrial and nuclear. The human mitochondrial genome is a tiny, circular, double-stranded, $16.6 \mathrm{~kb}$ DNA encoding 13 protein subunits of the respiratory chain complexes, as well as 2 ribosomal RNAs and 22 tRNAs essential for mitochondrial protein synthesis. ${ }^{1}$ More than $99 \%$ of the mitochondrial proteins are encoded by the nuclear genome. Mitochondrial biogenesis, respiratory chain complex assembly, mtDNA replication, transcription, and mitochondrial protein biosynthesis all require nuclear DNA-encoded factors. ${ }^{2-4}$ Accordingly, mitochondrial disorders are a complex dual genome disease that can be caused by molecular defects in both nuclear and mitochondrial genomes with the nuclear gene defects as the major cause.
Copyright @ 2012 by Thieme Medical Publishers, Inc., 333 Seventh Avenue, New York, NY 10001, USA. Tel: +1(212) 584-4662.
DOI http://dx.doi.org/ 10.1055/s-0032-1306387. ISSN 0271-8235. 
Historically, inherited leukoencephalopathies in children have mostly been attributed to lysosomal storage disorders or peroxisomal diseases. However, in recent years, white matter involvement has been increasingly recognized as a common feature in patients with mitochondrial disorders. ${ }^{5,6}$ Because the brain is a tissue of high energy demand, mitochondrial dysfunction plays an important role in the etiology of encephalopathy.

As mitochondrial respiratory chain deficiency is a multisystem disorder, the occurrence of white matter abnormalities has not been systematically evaluated. Bilateral symmetric abnormalities in basal ganglia and brainstem are usually the common magnetic resonance imaging (MRI) features in children with mitochondrial encephalopathies. A review of 110 children with mitochondrial disorders identified eight patients (7.3\%) with MRI consistent with a leukoencephalopathy. ${ }^{7}$ However, the diagnosis of mitochondrial disorders in this cohort of patients was not confirmed by molecular findings of causative mutations. A recent study of 30 patients with complex I deficiency and confirmed molecular defects in mtDNA (20 patients) or nuclear genes encoding complex I (nDNA, 10 patients) revealed that 5 of the 10 patients carrying nDNA mutations in complex I subunits exhibited leukoencephalopathy, while stroke-like lesions were observed in 8 out of 19 carrying mtDNA mutations and in none of the patients with nDNA mutations. ${ }^{7}$ Thus, the incidence of leukoencephalopathies in patients with mitochondrial disorders and carrying mutations in the nuclear encoded genes in this cohort is $~ 50 \%$. Of course, this incidence may vary with the age of the patients at the time of ascertainment. The prevalence of common mtDNA mutations in the general population has been estimated to be at least 1 in $5000 .{ }^{8} \mathrm{~A}$ recent study of mtDNA common mutations in newborns and their mothers suggested the possibility of a much higher rate. ${ }^{9}$

The major function of mitochondria is to produce energy to support various cellular activities. Therefore, the biochemical cause of encephalopathy is energy deficiency. The molecular etiology, however, is very heterogeneous. ${ }^{10}$ The disease may be caused by molecular defects in any of the $\sim 1,500$ mitochondrial targeting genes. The mode of inheritance may be autosomal recessive, autosomal dominant, $\mathrm{X}$-linked, or maternal. Mitochondrial disorders often have multisystem involvement. Encephalopathy is one of the more prevalent clinical features of the disorder. Leukoencephalopathy was first investigated in patients with molecular defects in the mitochondrial genome, including mtDNA point mutations and large deletions. ${ }^{11}$ With the recent discovery of nuclear genes responsible for mitochondrial respiratory chain defects, studies of Leigh's disease with white matter involvement caused by nuclear genes encoding complex subunits and assembly factors have been reported. ${ }^{6}$ In addition, leukoencephalopathies in mitochondrial syndromes caused by defects in a group of nuclear genes involving the maintenance of mtDNA integrity, such as DNA polymerase gamma (POLG), thymidine phosphorylase (TYMP), and MPV17 have also been reported. ${ }^{12-15}$ More recently, mutations in mitochondrial aspartyl-tRNA synthase have been found to be associated with leukoencephalopathy with brainstem and spinal cord involvement and lactate elevation (LBSL). ${ }^{16,} 17$ Clinical and MRI features of representative cases in these categories are described below.

\section{Clinical and MRI Features}

White matter abnormalities may be characteristic depending on the molecular etiology of the mitochondrial disorder. In general, lesions caused by mtDNA point mutations predominantly affect deep gray matter with minor peripheral white matter involvement. Diffuse supratentorial, most likely necrotizing leukoencephalopathies, involving the deep lobar white matter are more frequently observed in patients with Leigh's disease caused by mutations in nuclear genes encoding complex I or IV subunits and assembly factors. ${ }^{5,6}$

\section{Mitochondrial Leukoencephalopathy Caused By Mutations in mtDNA}

Mitochondrial Encephalomyopathy with Lactic Acidosis and Stroke-Like Episodes

Mitochondrial encephalomyopathy with lactic acidosis and stroke-like episodes (MELAS) is the most common maternally inherited multisystem disorder. The clinical spectrum is broad and may overlap with other syndromes. MELAS patients often have short stature and major symptoms, including generalized tonic-clonic seizures, recurrent migraine headaches, muscle weakness or exercise intolerance, and sensorineural hearing loss. Lactic acidosis and stroke-like episodes with hemiparesis and/or cortical blindness are the hallmarks. Early development is usually unremarkable. Clinical manifestations may occur during childhood or early adulthood. The molecular defects occur at the mtDNA mutation hot spot, tRNA ${ }^{\text {Leu(UUR) }}$ gene. The m.3243A > G mutation accounts for $\sim 80 \%$ of MELAS (http://www.mitomap.org). Mutations in other tRNA and mRNA genes causing MELAS have also been observed. Mitochondrial proliferation revealed by electron microscopy as enlarged or increased number of mitochondria is characteristic of tRNA mutations in mtDNA. Ragged red fibers in muscle biopsy are common in older patients, but usually are not obvious in young children. Brain MRI shows infarct-like lesions predominantly affecting the occipital cortex that usually do not conform to large vascular territories. Small nonspecific periventricular white matter abnormalities may occur. ${ }^{18} \mathrm{How}-$ ever, diffuse symmetric posterior leukoencephalopathy lacking stroke-like lesions is very rare. ${ }^{19}$

\section{Myoclonic Epilepsy with Ragged Red Fibers}

Myoclonic epilepsy with ragged red fibers (MERRF), a multisystem mitochondrial disorder, is characterized by myoclonus, generalized seizures, cerebellar ataxia, mental deterioration, muscle weakness, and sensorineural hearing loss. Cardiomyopathy may occur. Early development is usually normal. Variable onset and clinical severity are seen in children and adults. The m.8344A $>\mathrm{G}$ mutation in the mtDNA tRNALys gene accounts for $\sim 80 \%$ of the molecular defects of the reported MERRF patients. Neuroradiologic findings of MERRF patients have rarely been described. 
From the few early reports, the MRI findings include cerebral atrophy, cerebral white matter T2 hyperintensities, striatal T2 hyperintensities, pallidal atrophy, and cerebellar atrophy. Recent studies of three patients also showed brainstem and cerebellar abnormalities. ${ }^{20}$ Recently, cavitating leukoencephalopathy was reported in a child carrying the m.8344A > G mutation. $^{21}$

\section{Leber's Hereditary Optic Neuropathy}

Leber's hereditary optic neuropathy (LHON) is characterized by the acute or subacute onset of bilateral optic neuropathy during the second or third decade of life. The causative mutations are missense mutations in mitochondrial encoded complex I subunits. The most common mutations are $\mathrm{m} .11778 \mathrm{G}>\mathrm{A}, \mathrm{m} .3460 \mathrm{G}>\mathrm{A}$, and $\mathrm{m} .14484 \mathrm{~T}>\mathrm{C}$. Although neurologic symptoms other than the optic nerve are rare, periventricular white matter involvement associated with LHON has been reported. ${ }^{22-25}$ Furthermore, atypical presentation of Leigh's disease associated with LHON has also been described. ${ }^{26}$

\section{Leigh's Syndrome}

Leigh's syndrome is a multisystem neurodegenerative disorder, characterized by necrotizing encephalomyelopathy. Patients usually have infantile onset and early death. Episodes of decompensation, particularly associated with viral infections, result in developmental regression, hypotonia, spasticity, movement disorders, and ataxia. Cardiomyopathy may occur. The disease is caused by deficiencies in mitochondrial respiratory energy metabolism. Therefore, mutations in mtDNA and nuclear genes involving all five respiratory chain complexes potentially can cause Leigh's syndrome. The most common mutations in the mtDNA causing Leigh's syndrome are $\mathrm{m} .8993 \mathrm{~T}>\mathrm{G}$ and $\mathrm{T}>\mathrm{C}$ in the ATP synthase subunit, ATPase 6 , of complex $\mathrm{V}^{27}$ White matter involvement in Leigh's disease has also been reported to be associated with m.9176T $>\mathrm{C}^{28}$ m.13513G $>\mathrm{A},{ }^{29}$ m.14459G $>\mathrm{A},{ }^{30}$ and $\mathrm{m} .14724 \mathrm{G}>\mathrm{A} .{ }^{31}$ The majority of Leigh's syndrome cases are due to isolated complex I or IV deficiencies caused by nuclear gene encoded complex subunits or complex assembly factors (discussed below).

\section{Kearns-Sayre's syndrome}

Kearns-Sayre's (KSS) syndrome is diagnosed by the triad of (a) onset of a neurodegenerative disorder before the age of 20, (b) pigmentary retinopathy that may lead to vision loss, and (c) ophthalmoplegia and/or ptosis, with one of the following: cardiac conduction defect, cerebellar dysfunction, or elevated cerebrospinal fluid (CSF) protein $(>100 \mathrm{mg} / \mathrm{dL})$. This multisystem disorder is caused by heteroplasmic large deletions of the mtDNA. White matter abnormalities were first described in $1979^{32}$ by computed tomography (CT), and it was later shown by others ${ }^{33}$ that diffuse spongiform degeneration of the white matter in the cerebrum, brainstem, and cerebellum were due to splitting of myelin lamellae. Leukoencephalopathy in KSS has also been demonstrated by MRI that showed bilateral high-signal lesions in subcortical white matter, thalamus, and brainstem. The white matter lesions may extend into the deep cerebral white matter and also affect the cerebellum. ${ }^{6}$

\section{Mitochondrial Leukoencephalopathy Caused By Mutations in Nuclear Genes}

\section{Leigh's Syndrome}

As mentioned above, Leigh's syndrome is the result of energy deficiency with a heterogeneous genetic etiology. In addition to the mutations in mtDNA, molecular defects in many nuclear gene encoded proteins involving mitochondrial energy metabolism may also cause Leigh's syndrome. The most frequent causes are deficiencies in the pyruvate dehydrogenase complex (PDHC) and the respiratory chain complexes.

\section{Pyruvate Dehydrogenase Complex}

The clinical symptoms of patients with PDHC deficiency vary from severe neonatal lactic acidosis and early death to intermittent ataxia or progressive neurodegenerative disorder with mental retardation and congenital brain malformations. $^{34-36}$ As the most common defects are in the E1 $\alpha$ subunit located on the X-chromosome, clinical presentation differs between male and female patients, although both sexes are almost equally affected. ${ }^{36}$ Male patients mainly present with devastating severe lactic acidosis in the neonatal period, resulting in early death, or with seizures, developmental delay, microcephaly, brain atrophy, and dysgenesis of brain structure, including agenesis of corpus callosum. In general, clinical symptoms of female patients are milder with variable phenotype due to random X-inactivation. Neurologically, seizures, cerebellar ataxia, and spasticity of the extremities are common. MRI findings include abnormal signal intensity of the basal ganglia, cerebellar and cerebral atrophy, and anomaly of the corpus callosum.., 36

\section{Complex Subunits and Complex Assembly Genes}

Isolated deficiencies of respiratory chain complexes result in Leigh's syndrome and often have white matter involvement. The most common defects are in complex I. White matter involvement has been described in numerous patients with Leigh's syndrome and isolated complex I deficiency due to mutations in complex subunits or assembly genes. MRI scans reveal severe supratentorial white matter changes and increased signal of the atrophic cerebellar cortex without significant involvement of basal ganglia or brainstem.6, 37, 38 Progressive hypodensity of the basal ganglia and midbrain has been reported. ${ }^{39}$ In addition to white-matter involvement, optic atrophy and hypertrophic cardiomyopathy may be present in some patients. ${ }^{39,40}$ Severe atrophy of both gray and white matter with demyelination and subventricular cysts have also been reported. ${ }^{41}$ A recent article described progressive cavitating leukoencephalopathy with multiple cysts involving the white matter, corpus callosum, and brainstem, with sparing of subcortical white matter and basal ganglia. ${ }^{42}$

Patients with complex II deficiency also present with typical Leigh's syndrome. ${ }^{43}, 44$ Brain MR spectroscopy (MRS) demonstrates prominent lactate and succinate peaks in the white matter and MRI shows severe cerebral and 
cerebellar leukodystrophy with or without basal ganglia involvement. $^{43,45}$

White matter lesions and basal ganglia calcifications have also been observed in two patients with complex III deficiency. ${ }^{46,47}$ The MRI of one patient showed high signal intensity in the temporoparietal white matter, while the other had dementia caused by subcortical leukodystrophy.

Complex IV deficiency is the second most common isolated complex deficiency causing Leigh's syndrome. The most common cause of isolated complex IV deficiency is due to the molecular defects in the SURF1 gene, which is responsible for the assembly of complex IV. ${ }^{48}$ Timothy and Geller in 2009 reported an infant boy with SURF1 mutation and diffuse periventricular white matter involvement, adding to the growing list of mitochondrial leukoencephalopathy. ${ }^{49}$

Leukoencephalopathy associated with complex $\mathrm{V}$ deficiency due to nuclear gene defects has not been reported.

\section{Genes Involved in the Maintenance of mtDNA Integrity}

A group of nuclear encoded genes responsible for mtDNA synthesis and/or maintenance of mtDNA integrity have recently been reported to cause mtDNA depletion and multiple deletions. ${ }^{4}$ Depending on the defective genes, there are hepatocerebral, myopathic, encephalomyopathic, and neurogastrointestinal encephalomyopathic forms of mtDNA depletion syndromes. ${ }^{4}$ White matter involvement has been described in patients with mutations in at least three genes: thymidine phosphorylase (TYMP), POLG, and MPV17.

\section{Mitochondrial Neurogastrointestinal Encephalomyopathy} Mitochondrial neurogastrointestinal encephalomyopathy (MNGIE) was the first reported syndrome that demonstrated the nuclear/mitochondrial cross-talk defect resulting in mtDNA depletion, which is secondary to primary defects in the nuclear gene, TYMP. ${ }^{13,14}$ The age of onset varies from 5 months to 43 years. The most common neurologic symptoms of MNGIE are peripheral neuropathy, ptosis, ophthalmoparesis, and hearing loss. Gastrointestinal dysmotility is the most prominent manifestation with recurrent diarrhea and intestinal pseudoobstruction. White matter involvement is the hallmark of MNGIE. All MNGIE patients who underwent MRI showed diffuse cerebral leukodystrophy. In some patients, the corpus callosum was relatively spared. There are reports of MNGIE-like patients who exhibited neurogastrointestinal encephalomyopathy without leukodystrophy. These are MNGIE-like patients who do not carry TYMP mutations, but rather mutations in other nuclear genes, such as POLG and RRM2B. ${ }^{50,51}$

\section{Alpers Syndrome and POLG-Related Disorders}

The most common nuclear gene defects that cause mitochondrial disorders are mutations in the POLG gene. POLG is the only DNA polymerase responsible for mtDNA biogenesis. Defects in POLG can cause autosomal dominant or autosomal recessive disorders. ${ }^{15}$ Alpers syndrome (OMIM\#203700) is the most common autosomal recessive disease of POLGrelated disorders, characterized by intractable seizures, ear- ly-onset progressive liver disease, and psychomotor regression. ${ }^{52}$ Kollberg and coworkers described the progressive neurodegenerative disorder in seven Alpers syndrome patients ages 4 months to 11 years. ${ }^{53}$ Brain MRI revealed progressive multifocal lesions predominantly affecting the gray matter of the supratentorial region, especially the parietal or occipital lobes. Lesions in white matter were observed in a couple of patients. Neurologic deterioration may be rapidly progressive as described by Brunetti-Pierri et $\mathrm{al}^{54}$ such that the normal brain MRI of an 8-month old patient showed a marked change in 5 weeks to diffuse cerebral atrophy affecting both gray and white matter. ${ }^{54}$ Progressive prominent white matter involvement has been reported in a 10 -year-old girl. ${ }^{55}$ The MRI taken at 3,7 , and 10 months after the disease onset showed striking progressive abnormalities of white matter in both occipital regions of diffuse brain atrophy and extensive abnormal signal in white matter. ${ }^{55}$

POLG mutations also cause multisystem mitochondrial disorders associated with progressive external ophthalmoplegia, ptosis, myopathy, and hearing loss. ${ }^{15,56}$ Visual symptoms associated with POLG-related disorders have been reported. $^{57}$ The MRI of the patients with visual loss showed volume loss of the optic nerves and chiasm. In addition, mild generalized brain atrophy and subcortical/ periventricular white matter signal abnormalities were also observed. $^{57}$

\section{Navajo Neurohepatopathy}

Navajo neurohepatopathy ( $\mathrm{NNH}$ ) is an autosomal recessive multisystem disorder prevalent in the Navajo population of the southwestern United States with an estimated incidence of 1 in 1,600 live births, but is very rare in the general population. ${ }^{58}$ The clinical features of the infantile and childhood forms of NNH are dominated by severe hepatopathy and early death from liver failure. ${ }^{59}$ The classic form of NNH is characterized by moderate liver disease and progressive neuropathy. Leukoencephalopathy with cerebral or cerebellar white matter involvement is present in almost all patients; however, the neurologic symptoms may not have surfaced when patients with the infantile hepatic form die in infancy. ${ }^{60}$ $\mathrm{NNH}$ is caused by mutations in the MPV17 gene encoding a mitochondrial inner membrane protein of unclear function, but its deficiency results in severe mtDNA depletion in the liver. ${ }^{61}$ Diffuse white matter hyperintense lesions involving posterior periventricular, subcortical, and cerebellar regions have been observed. ${ }^{59-62}$

Other Genes Causing mtDNA Depletion Or mtDNA Integrity Maintenance-Associated Encephalopathy

Mutations in at least 10 genes are known to cause mtDNA depletion or multiple deletion syndromes, and most of these are associated with encephalopathy. ${ }^{10}$ White matter involvement is variable. ${ }^{50}$ In one recent report, leukoencephalopathy was detected prenatally in an infant with congenital lactic acidosis that was suggested by fetal MRI showing periventricular hyperintense lesions. ${ }^{63}$ Deleterious mutations were identified in the SUCLG1 gene. ${ }^{63}$ 
Leukoencephalopathy with Brainstem and Spinal Cord Involvement and Lactate Elevation

Leukoencephalopathy with brainstem and spinal cord involvement and lactate elevation (LBSL) is a recently identified multisystem disease caused by mutations in the DARS2 gene encoding the mitochondrial aspartyl-tRNA synthetase, one of the 20 aminoacyl-tRNA synthetases in mitochondria. ${ }^{16}$ The disease has childhood onset with slowly progressive pyramidal, cerebellar, and dorsal column dysfunction. ${ }^{17,}$ 64-66 The MRI shows signal abnormalities in the cerebral and cerebellar white matter and specific brainstem and spinal cord tracts. ${ }^{16}$, 17 This unique MRI pattern differs from classic and other recently defined leukoencephalopathies and is therefore diagnostic. However, MRI findings may vary during disease course, and precede motor symptoms by several years. ${ }^{64}$ Furthermore, the severity of MRI changes do not correlate with the severity of the clinical course, suggesting that although the MRI of LBSL is highly specific and diagnostic it does not necessarily reflect the clinical activity. ${ }^{66}$ Although LBSL is typical of patients with DARS2 mutations, lactate levels in the affected white matter are not always elevated. ${ }^{65,66}$

\section{Diagnosis}

Making and confirming the diagnosis of a specific mitochondrial multisystem disorder is a great challenge due to the extreme genetic and clinical heterogeneity. ${ }^{67-69}$ Although there are $\sim 1,500$ genes reported that are potentially responsible for mitochondrial disorders, biochemical analyses, neuroradiologic studies, and clinical features may help narrow down the disease to a group of causative genes for further analyses, before reaching the ultimate molecular diagnosis of the disorder.

\section{Biochemical Markers}

\section{Blood and CSF Analytes}

Elevation of lactate in blood or CSF is an important marker of mitochondrial disease, although not specific. Measurement of lactate/pyruvate ratios, which indirectly reflects the NADH/ $\mathrm{NAD}^{+}$redox status, may be necessary. Pyruvate is another useful marker. The lactate/pyruvate ratio increases if there is a respiratory chain defect. However, if there is a deficiency in pyruvate metabolism, such as pyruvate dehydrogenase complex or pyruvate carboxylase, then plasma or CSF levels of pyruvate will elevate. Elevated plasma alanine is a useful indicator of long-standing pyruvate accumulation. It is important to note that mitochondrial disease patients with predominantly brain manifestations may have elevated levels of CSF lactate or pyruvate without an elevation of blood lactate. Therefore, using MRS to detect elevations of lactate or pyruvate in specific brain regions is a more diagnostic approach. For complex II deficiency, accumulation of succinate is an indicator.

Other biochemical markers include elevated plasma tyrosine, phenylalanine, or methionine suggesting liver disease; abnormal urine organic acids such as 3-methyl glutaconic acid or dicarboxylic acids, suggesting mitochondrial dysfunc- tion; or elevation of acylcarnitine, suggesting defects in mitochondrial fatty acid oxidation. These are all indications of multisystem mitochondrial disease association.

\section{Electron Transport Chain Complex Activities}

If muscle biopsies are available, the mitochondrial functions including electron transport chain (ETC) activities, ATP synthesis, or oxygen consumption rate can be assessed to support the presence of mitochondrial dysfunction. Skin fibroblast cultures are useful in the detection of isolated complex deficiencies in Leigh's disease if the enzyme activities are expressed in the skin fibroblasts. The results of ETC analyses will indicate which complex is causing the isolated deficiency, or if there is a more generalized complex deficiency suggestive of mtDNA depletion syndrome, tRNA defects, or mitochondrial protein translation defects, hence assisting in narrowing down the number of candidate genes to be analyzed molecularly. Because ETC deficiency may be tissue specific, a normal ETC does not rule out the diagnosis of mitochondrial disorders.

\section{Magnetic Resonance Imaging and Magnetic Resonance Spectroscopy}

MRI is a valuable tool in the evaluation of anatomic structures in neurometabolic disorders, such as mitochondrial syndromes. In the previous section, specific MRI features in each mitochondrial syndrome were described. However, MRI findings for mitochondrial disorders may be nonspecific or may change over time. MRS detects the unique proton resonance frequency called chemical shift, from the specific metabolic compound. Therefore, the MRS peaks of lactate, pyruvate, succinate, myoinositol, creatine, and $\mathrm{N}$-acetyl-1aspartate, which provide rough estimates of the abnormal metabolite concentration in different regions of the brain, can help with the diagnosis. ${ }^{68}$

\section{Clinical Diagnosis}

As described above, due to the broad heterogeneity and extensive overlap of clinical features among various multisystem mitochondrial syndromes, based on the affected organs or tissues, clinical diagnosis of mitochondrial disorders may at best classify the disease into categories of recognizable syndromes, such as hepatocerebral, encephalomyopathic, myopathic, Leigh's, MELAS, PEO, or KSS. A group of candidate genes responsible for the associated symptoms may be indicated. Nevertheless, the ultimate confirmatory diagnosis relies on the identification of causative mutations in the responsible gene. ${ }^{69}$

\section{Indication for Diagnosis of Mitochondrial Disorders and Differentials}

Mitochondrial respiratory chain disorders predominantly affect the central nervous system (CNS) and are frequent causes of diffuse leukoencephalopathy. Thorough evaluation for mitochondrial disorders, including blood and CSF biochemical markers, MRS and MRI, is warranted in patients with complex neurologic features and multisystem involvement. Patients with autosomal recessive mutations in nuclear 
genes usually have a more severe clinical course and present significantly earlier with brain anomalies than patients with mtDNA mutations. Stroke-like lesions are frequent and only observed in patients with mtDNA mutations, but not in patients with PDHC deficiency. Supratentorial stroke-like lesions may also be seen in patients with POLG mutations. Anomaly of the corpus callosum is very frequent in $\mathrm{PDH}$ deficient patients but not observed in complex I deficient patients. Patients with complex I deficiency and necrotizing leukoencephalopathy are likely to have mutations in nuclear encoded genes. Diffuse periventricular white matter abnormalities are usually associated with mutations in nuclear genes, including the genes causing mtDNA depletion syndrome.

\section{Ultimate Definitive Molecular Diagnosis}

The ultimate definitive diagnosis of mitochondrial disorders is by way of identifying the deleterious mutations in the causative genes. Due to the large number of genes and the complex dual genome involvement, definitive diagnosis is difficult. The current recommendation for the diagnostic algorithm of mitochondrial disorders is first screening of common mtDNA point mutations (e.g., MELAS) and large deletions (e.g., KSS) followed by direct sequencing of the specific causative genes for recognizable syndromes. ${ }^{69}$ If maternal inheritance pattern is indicated, the whole mitochondrial genome should be sequenced. With the recent development of massive parallel sequencing of the enriched mitochondrial targeting genes, the entire 1500 genes plus the whole mitochondrial genome can be analyzed simultaneously by the next generation sequencing approach. ${ }^{70}$

\section{References}

1 Wallace DC, Lott MT, Brown MD, Kerstann K. Mitochondria and neuro-ophthalmologic diseases. In: Scriver CR, Beaudet AL, Sly WS, Valle D, eds. The Metabolic \& Molecular Bases of Inherited Disease. Vol II. 8th ed. New York: McGraw-Hill; 2001 2425-2509

2 Koene S, Smeitink J. Mitochondrial medicine: entering the era of treatment. J Intern Med 2009;265(2):193-209

3 Pagliarini DJ, Calvo SE, Chang B, et al. A mitochondrial protein compendium elucidates complex I disease biology. Cell 2008;134 (1):112-123

4 Spinazzola A, Zeviani M. Disorders of nuclear-mitochondrial intergenomic signaling. Gene 2005;354:162-168

5 Lebre AS, Rio M, Faivre d'Arcier L, et al. A common pattern of brain MRI imaging in mitochondrial diseases with complex I deficiency.J Med Genet 2011;48(1):16-23

6 Lerman-Sagie T, Leshinsky-Silver E, Watemberg N, Luckman Y, Lev D. White matter involvement in mitochondrial diseases. Mol Genet Metab 2005;84(2):127-136

7 Moroni I, Bugiani M, Bizzi A, Castelli G, Lamantea E, Uziel G. Cerebral white matter involvement in children with mitochondrial encephalopathies. Neuropediatrics 2002;33(2):79-85

8 Schaefer AM, Taylor RW, Turnbull DM, Chinnery PF. The epidemiology of mitochondrial disorders-past, present and future. Biochim Biophys Acta 2004;1659(2-3):115-120

9 Elliott HR, Samuels DC, Eden JA, Relton CL, Chinnery PF. Pathogenic mitochondrial DNA mutations are common in the general population. Am J Hum Genet 2008;83(2):254-260
10 Wong LJ. Molecular genetics of mitochondrial disorders. Dev Disabil Res Rev 2010;16(2):154-162

11 de Lonlay-Debeney P, von Kleist-Retzow JC, Hertz-Pannier L, et al. Cerebral white matter disease in children may be caused by mitochondrial respiratory chain deficiency. J Pediatr 2000;136 (2):209-214

12 El-Hattab AW, Li FY, Schmitt E, Zhang S, Craigen WJ, Wong LJ. MPV17-associated hepatocerebral mitochondrial DNA depletion syndrome: new patients and novel mutations. Mol Genet Metab 2010;99(3):300-308

13 Nishino I, Spinazzola A, Hirano M. Thymidine phosphorylase gene mutations in MNGIE, a human mitochondrial disorder. Science 1999;283(5402):689-692

14 Nishino I, Spinazzola A, Papadimitriou A, et al. Mitochondrial neurogastrointestinal encephalomyopathy: an autosomal recessive disorder due to thymidine phosphorylase mutations. Ann Neurol 2000;47(6):792-800

15 Tang S, Wang J, Lee NC, et al. Mitochondrial DNA polymerase gamma mutations: an ever expanding molecular and clinical spectrum. J Med Genet 2011;48(10):669-681

16 Scheper GC, van der Klok T, van Andel RJ, et al. Mitochondrial aspartyl-tRNA synthetase deficiency causes leukoencephalopathy with brain stem and spinal cord involvement and lactate elevation. Nat Genet 2007;39(4):534-539

17 Uluc K, Baskan O, Yildirim KA, et al. Leukoencephalopathy with brain stem and spinal cord involvement and high lactate: a genetically proven case with distinct MRI findings. J Neurol Sci 2008;273(1-2):118-122

18 Castillo M, Kwock L, Green C. MELAS syndrome: imaging and proton MR spectroscopic findings. AJNR Am J Neuroradiol 1995;16 (2):233-239

19 Renard D, Bonnaure H, Labauge P. Teaching NeuroImages: diffuse posterior leukoencephalopathy in MELAS without stroke-like episodes. Neurology 2010;75(3):e9

20 Ito S, Shirai W, Asahina M, Hattori T. Clinical and brain MR imaging features focusing on the brain stem and cerebellum in patients with myoclonic epilepsy with ragged-red fibers due to mitochondrial A8344G mutation. AJNR Am J Neuroradiol 2008;29(2): 392-395

21 Biancheri R, Rossi D, Cassandrini D, Rossi A, Bruno C, Santorelli FM. Cavitating leukoencephalopathy in a child carrying the mitochondrial A8344G mutation. AJNR Am J Neuroradiol 2010;31(9): E78-E79

22 Bhatti MT, Newman NJ. A multiple sclerosis-like illness in a man harboring the mtDNA 14484 mutation. J Neuroophthalmol 1999;19(1):28-33

23 Jansen PH, van der Knaap MS, de Coo IF. Leber's hereditary optic neuropathy with the 11778 mtDNA mutation and white matter disease resembling multiple sclerosis: clinical, MRI and MRS findings. J Neurol Sci 1996;135(2):176-180

24 Lev D, Yanoov-Sharav M, Watemberg N, Leshinsky-Silver E, Lerman-Sagie T. White matter abnormalities in Leber's hereditary optic neuropathy due to the 3460 mitochondrial DNA mutation. Eur J Paediatr Neurol 2002;6(2):121-123

25 Nikoskelainen EK, Marttila RJ, Huoponen K, et al. Leber's "plus": neurological abnormalities in patients with Leber's hereditary optic neuropathy. J Neurol Neurosurg Psychiatry 1995;59 (2):160-164

26 Fruhman G, Landsverk ML, Lotze TE, et al. Atypical presentation of Leigh syndrome associated with a Leber hereditary optic neuropathy primary mitochondrial DNA mutation. Mol Genet Metab 2011;103(2):153-160

27 Enns GM, Bai RK, Beck AE, Wong LJ. Molecular-clinical correlations in a family with variable tissue mitochondrial DNA T8993G mutant load. Mol Genet Metab 2006;88(4):364-371

28 Hung PC, Wang HS. A previously undescribed leukodystrophy in Leigh syndrome associated with T9176C mutation of the mitochondrial ATPase 6 gene. Dev Med Child Neurol 2007;49(1):65-67 
29 Brautbar A, Wang J, Abdenur JE, et al. The mitochondrial $13513 \mathrm{G}$ $>$ A mutation is associated with Leigh disease phenotypes independent of complex I deficiency in muscle. Mol Genet Metab 2008;94(4):485-490

30 Gropman A, Chen TJ, Perng CL, et al. Variable clinical manifestation of homoplasmic G14459A mitochondrial DNA mutation. Am J Med Genet A 2004;124A(4):377-382

31 Pereira C, Nogueira C, Barbot C, et al. Identification of a new mtDNA mutation (14724G $>$ A) associated with mitochondrial leukoencephalopathy. Biochem Biophys Res Commun 2007;354(4):937-941

32 Seigel RS, Seeger JF, Gabrielsen TO, Allen RJ. Computed tomography in oculocraniosomatic disease (Kearns-Sayre syndrome). Radiology 1979;130(1):159-164

33 Oldfors A, Fyhr IM, Holme E, Larsson NG, Tulinius M. Neuropathology in Kearns-Sayre syndrome. Acta Neuropathol 1990;80 (5):541-546

34 Brown GK, Otero LJ, LeGris M, Brown RM. Pyruvate dehydrogenase deficiency. J Med Genet 1994;31(11):875-879

35 Dahl HH, Brown GK. Pyruvate dehydrogenase deficiency in a male caused by a point mutation (F205L) in the E1 alpha subunit. Hum Mutat 1994;3(2):152-155

36 Willemsen M, Rodenburg RJ, Teszas A, van den Heuvel L, Kosztolanyi G, Morava E. Females with PDHA1 gene mutations: a diagnostic challenge. Mitochondrion 2006;6(3):155-159

37 Loeffen JL, Smeitink JA, Trijbels JM, et al. Isolated complex I deficiency in children: clinical, biochemical and genetic aspects. Hum Mutat 2000;15(2):123-134

38 Schuelke M, Smeitink J, Mariman E, et al. Mutant NDUFV1 subunit of mitochondrial complex I causes leukodystrophy and myoclonic epilepsy. Nat Genet 1999;21(3):260-261

39 Loeffen J, Elpeleg O, Smeitink J, et al. Mutations in the complex I NDUFS2 gene of patients with cardiomyopathy and encephalomyopathy. Ann Neurol 2001;49(2):195-201

40 Ogilvie I, Kennaway NG, Shoubridge EA. A molecular chaperone for mitochondrial complex I assembly is mutated in a progressive encephalopathy. J Clin Invest 2005;115(10):2784-2792

41 Saada A, Edvardson S, Rapoport M, et al. C6ORF66 is an assembly factor of mitochondrial complex I. Am J Hum Genet 2008;82 (1):32-38

42 Ferreira M, Torraco A, Rizza T, et al. Progressive cavitating leukoencephalopathy associated with respiratory chain complex I deficiency and a novel mutation in NDUFS1. Neurogenetics 2011;12(1):9-17

43 Brockmann K, Bjornstad A, Dechent P, et al. Succinate in dystrophic white matter: a proton magnetic resonance spectroscopy finding characteristic for complex II deficiency. Ann Neurol 2002;52 (1):38-46

44 Burgeois M, Goutieres F, Chretien D, Rustin P, Munnich A, Aicardi J. Deficiency in complex II of the respiratory chain, presenting as a leukodystrophy in two sisters with Leigh syndrome. Brain Dev 1992;14(6):404-408

45 Ghezzi D, Goffrini P, Uziel G, et al. SDHAF1, encoding a LYR complex-II specific assembly factor, is mutated in SDH-defective infantile leukoencephalopathy. Nat Genet 2009;41(6):654656

46 Majoie CB, Akkerman EM, Blank C, Barth PG, Poll-The BT, den Heeten GJ. Mitochondrial encephalomyopathy: comparison of conventional MR imaging with diffusion-weighted and diffusion tensor imaging: case report. AJNR Am J Neuroradiol 2002;23(5):813-816

47 Seijo-Martínez M, Castro del Río M, Campos Y, et al. Unusual clinical findings and Complex III deficiency in a family with myotonic dystrophy. J Neurol Sci 2003;208(1-2):87-91

48 Rahman S, Brown RM, Chong WK, Wilson CJ, Brown GKA. A SURF1 gene mutation presenting as isolated leukodystrophy. Ann Neurol 2001;49(6):797-800

49 Timothy J, Geller T. SURF-1 gene mutation associated with leukoencephalopathy in a 2-year-old. J Child Neurol 2009;24 (10):1296-1301
50 Shaibani A, Shchelochkov OA, Zhang S, et al. Mitochondrial neurogastrointestinal encephalopathy due to mutations in RRM2B. Arch Neurol 2009;66(8):1028-1032

51 Tang S, Dimberg EL, Milone M, Wong LJ. Mitochondrial neurogastrointestinal encephalomyopathy (MNGIE)-like phenotype: an expanded clinical spectrum of POLG1 mutations. J Neurol 2011

52 Harding BN. Progressive neuronal degeneration of childhood with liver disease (Alpers-Huttenlocher syndrome): a personal review. J Child Neurol 1990;5(4):273-287

53 Kollberg G, Moslemi AR, Darin N, et al. POLG1 mutations associated with progressive encephalopathy in childhood. J Neuropathol Exp Neurol 2006;65(8):758-768

54 Brunetti-Pierri N, Selby K, O'Sullivan M, et al. Rapidly progressive neurological deterioration in a child with Alpers syndrome exhibiting a previously unremarkable brain MRI. Neuropediatrics 2008;39(3):179-183

55 Bao X, Wu Y, Wong LJ, et al. Alpers syndrome with prominent white matter changes. Brain Dev 2008;30(4):295-300

56 Wong LJ, Naviaux RK, Brunetti-Pierri N, et al. Molecular and clinical genetics of mitochondrial diseases due to POLG mutations. Hum Mutat 2008;29(9):E150-E172

57 Milone M, Wang J, Liewluck T, Chen LC, Leavitt JA, Wong LJ. Novel POLG splice site mutation and optic atrophy. Arch Neurol 2011;68 (6):806-811

58 Holve S, Hu D, Shub M, Tyson RW, Sokol RJ. Liver disease in Navajo neuropathy. J Pediatr 1999;135(4):482-493

59 Karadimas CL, Vu TH, Holve SA, et al. Navajo neurohepatopathy is caused by a mutation in the MPV17 gene. Am J Hum Genet 2006;79 (3):544-548

60 Wong LJ, Brunetti-Pierri N, Zhang Q et al. Mutations in the MPV17 gene are responsible for rapidly progressive liver failure in infancy. Hepatology 2007;46(4):1218-1227

61 Spinazzola A, Viscomi C, Fernandez-Vizarra E, et al. MPV17 encodes an inner mitochondrial membrane protein and is mutated in infantile hepatic mitochondrial DNA depletion. Nat Genet 2006;38 (5):570-575

62 Navarro-Sastre A, Martín-Hernández E, Campos Y, et al. Lethal hepatopathy and leukodystrophy caused by a novel mutation in MPV17 gene: description of an alternative MPV17 spliced form. Mol Genet Metab 2008;94(2):234-239

63 Randolph LM, Jackson HA, Wang J, et al. Fatal infantile lactic acidosis and a novel homozygous mutation in the SUCLG1 gene: a mitochondrial DNA depletion disorder. Mol Genet Metab 2011;102(2):149-152

64 Isohanni P, Linnankivi T, Buzkova J, et al. DARS2 mutations in mitochondrial leucoencephalopathy and multiple sclerosis. J Med Genet 2010;47(1):66-70

65 Mierzewska H, van der Knaap MS, Scheper GC, BekiesinskaFigatowska M, Szczepanik E, Jurkiewicz E. Leukoencephalopathy with brain stem and spinal cord involvement and lactate elevation in the first Polish patient. Brain Dev 2011;33(9):713-717

66 Tzoulis C, Tran GT, Gjerde IO, et al.Leukoencephalopathy with brainstem and spinal cord involvement caused by a novel mutation in the DARS2 gene. J Neurol 2011

67 Haas RH, Parikh S, Falk MJ, et al. Mitochondrial disease: a practical approach for primary care physicians. Pediatrics 2007;120 (6):1326-1333

68 Haas RH, Parikh S, Falk MJ, et al; Mitochondrial Medicine Society's Committee on Diagnosis. The in-depth evaluation of suspected mitochondrial disease. Mol Genet Metab 2008;94(1): $16-37$

69 Wong LJ, Scaglia F, Graham BH, Craigen WJ. Current molecular diagnostic algorithm for mitochondrial disorders. Mol Genet Metab 2010;100(2):111-117

70 Vasta V, Ng SB, Turner EH, Shendure J, Hahn SH. Next generation sequence analysis for mitochondrial disorders. Genome Med 2009;1(10):100 